\title{
LA CONCILIACIÓN FAMILIAR Y LABORAL EN ESPAÑA EN LA ESFERA POLÍTICA, MEDIÁTICA Y EMPRESARIAL. UN ESTUDIO DE AGENDAS ENTRE 2003 Y 2007
}

\author{
Marta Martín Llaguno \\ marta.martin@ua.es \\ Universidad de Alicante
}

\author{
Consuelo León Llorente \\ cleonllorente@gmail.com \\ Universitat Internacional de Catalunya
}

Cristina Guirao Mirón

cguirao@um.es

Universidad de Murcia

Recibido: 28-02-2013

Aceptado: 17-03-2013

\section{Resumen}

Desde que se publica la Ley 39/99, impelida desde la UE, en España la conciliación familiar y laboral se va a incorporando a la esfera pública como asunto de interés. Como sucede en otros países, al tiempo que se produce el crecimiento de las tasas de empleo femenino se inicia un debate para la solución de este problema. Este trabajo explora su protagonismo en tres esferas la legislativa, la periodística y la empresarial- entre el año 2003 (IV Plan para la Igualdad) y el 2007 (promulgación de la Ley Orgánica 3/2007 - LOIEMH), en el periodo del "milagro económico español”. A partir de la recopilación de todas las iniciativas parlamentarias del Congreso de los Diputados, las informaciones publicadas en los tres diarios nacionales de mayor tirada (El Mundo, El País y ABC) y de las repuestas de directivos de 4700 empresas en el estudio IFREI, se revisa la dinámica de la construcción de la conciliación como tema social.

Palabras Clave: Conciliación vida familiar y laboral, esfera pública, iniciativas parlamentarias, congreso de los diputados, cobertura mediática.

\begin{abstract}
Since the Law 39/99 is published, propelled by the EU, work and family conflict becomes a public issue in Spain. As in other countries, while growth in female employment occurs, a debate to solve this social problem begins. This paper explores three areas -the legislative, the journalistic and the business one- between 2003 (IV Plan for Equality) and 2007 (when the Organic Law 3/2007 - LOIEMH entry into force), in the period of "Spanish economic miracle". From the collection of all parliamentary initiatives of the Congress of Deputies, the information published in the three largest circulation national newspapers (El Mundo, El Pais and $A B C$ ) and from the responses of managers of 4700 companies in the study IFREI, the dynamics of the social construction of the problem is reviewed.
\end{abstract}

Keywords: work-life balance, public sphere, parliamentary initiatives, Congress, media coverage. 


\section{Introducción}

Desde la década de los 80 van apareciendo en las sociedades del bienestar, amparadas inicialmente bajo el paraguas de la igualdad, políticas de protección de la maternidad que son superadas en los 90 por las llamadas políticas de conciliación. Estas políticas de conciliación, auspiciadas por los poderes públicos, tienen entre otros objetivos, la sensibilización hacia el tema de la opinión pública, porque hay bases culturales profundas que es preciso cambiar.

Como indican Fernández y Tobío (1999), es a mediados de los 90 del siglo XX cuando la conciliación entra en la agenda española como tema de preocupación social. Primero, en el marco de los planes comunitarios de igualdad de oportunidades, después, en el contexto de la preocupación por el envejecimiento de la población europea y la necesidad de incrementar la población activa femenina. En muy poco tiempo, España empieza a construir todo un marco jurídico relacionado con esta cuestión que refleja la preocupación sobre este tema y sobre su relación con la igualdad. Así, en noviembre de 1999 se promulga la Ley 39/1999, para promover la Conciliación de la Vida Familiar y Laboral de las personas trabajadoras (LCVFL). La aparición de esta norma hay que entenderla en el contexto de la obligación que nuestro país tiene de trasponer urgentemente dos directivas europeas: la $92 / 85$, de 19 de octubre, sobre salud y seguridad en el trabajo de la trabajadora embarazada, que haya dado a luz o en periodo de lactancia y la $96 / 34$, de 3 de junio, sobre el permiso parental que establece el disfrute indistinto entre hombres y mujeres de los permisos parentales. La ley traduce, no obstante, un inicio en la voluntad política y sindical de cambiar una situación de incompatibilidad entre la esfera laboral y familiar, sobre la que, todavía se sabe poco. En efecto, a partir de esos momentos, se despierta también un interés sociológico por el tema y se empiezan a llevar a cabo estudios importantes por sus conclusiones y por la formulación de ciertas cuestiones.

Estos estudios inciden, especialmente, en los sujetos pacientes de la conciliación, los hijos (Meil, 1997). Abordando la conciliación desde el vacío legal (Iglesias de Ussel, 1995), o centrándose en la masiva incorporación de la mujer al mercado laboral y sus consecuencias en el reparto de tareas (Del Campo, 1990). Entre los estudios académicos destacan los de Durán (2003) e Iglesias de Ussel (2004) y, más tarde, Tobío (2005) y Aguirre y García Sáinz (2005). También cabe mencionar trabajos como los de Meil (1997) y Villafañe Asociados (2005). En la misma línea y desde las estadísticas oficiales, las encuestas del INE centran en la cuestión de uso y reparto del tiempo en el ámbito doméstico (Durán, 1997) así como en el análisis de roles y tareas en el hogar. Respecto a la situación de las empresas y las palancas internas de cambio, de gran utilidad, 
especialmente en el diseño de variables, resultan las publicaciones sobre flexibilidad y usos del tiempo en la empresa de la mano de los programas Equal ${ }^{1}$ de la Unión Europea.

En el contexto de esta última literatura académica, el éxito de los programas ha sido una cuestión central (Beer et al. 1990); (Duck, 1993); (Clarke, 1994); (Vandermerwe y Vandermarwe, 1991). Los estudios dejan constancia de que más allá de razones de eficiencia productiva, las organizaciones tienden a la imitación de buenas prácticas (Meyer y Rowan, 1977) y a decidir influidos por las presiones legales del entorno. En efecto, Covin \& Kilmann (1990) identifican una serie de factores de éxito de los programas de conciliación empresarial que luego aparecen en el trabajo de Kraut (1990), quien investiga en seis multinacionales pioneras en este tema: Dupont, Merck, IBM, Bellsouth, Honeywell \& Mobil.

En el análisis de los programas de conciliación organizacionales en España, destaca el trabajo realizado por IESE a través de su índice IFREI (2003, 2005, 2007, 2009) y sus estudios derivados (Poelmans, Chinchilla y Cardona, 2003); (Chinchilla y León, 2005 y 2011); (León, 2010); (Chinchilla et al., 2007). Planteado desde una vertiente múltiple: desde la investigación académica, desde el foro de debate empresarial y desde la sensibilización de la opinión pública; el estudio IFREI, del IESE, es el primero que aborda la medición no sólo de las políticas disponibles en las instituciones, sino también de la cultura empresarial. Su cuestionario inicial presenta una lista de treinta y una políticas de conciliación y, además, evalúa aspectos facilitadores para su implantación como es el caso del compromiso directivo con el plan, el presupuesto asignado, la comunicación interna y el equipo responsable de llevarlo a cabo. También se pregunta acerca de la cultura de la empresa para analizar la resistencia a la flexibilidad dentro de las organizaciones: hábitos horarios, criterios en la promoción (igualdad) y sentido del compromiso (confianza). El cuestionario pasa de 158 preguntas (Chinchilla, Poelmans y León, 2003; Poelmans, Chinchilla y Cardona, 2003-2003a) a 63 preguntas y finalmente a 45 preguntas (Chinchilla, Núria; et al., 2007).

Los informes IFREI ${ }^{2}$, realizados en los últimos diez años sobre una muestra total de 4.700 empresas (León, 2010), confirman que las leyes, las iniciativas parlamentarias, los convenios y en definitiva el marco legal, hacen su aparición en entornos laborales con bajos niveles de flexibilidad y culturas poco igualitarias (Sánchez, Mayo y Chinchilla, 2006). De hecho, las políticas de flexibilidad en el espacio y el tiempo son las más escasas y a la vez las más demandadas por el empleado. Por otra parte, los IFREI señalan que el

\footnotetext{
${ }^{1}$ Véase: http://www.ideasbank-equal.info. Son miembros de la Asociación de Desarrollo de un Equal distintas administraciones públicas autonómicas y locales, agentes sociales: organizaciones sindicales y empresariales; y asociaciones sin ánimo de lucro. La Iniciativa Comunitaria EQUAL pretende ser un campo de pruebas para desarrollar y difundir nuevas maneras de aplicar las políticas sociales, principalmente las de empleo, a fin de combatir todo tipo de discriminación y desigualdad sufrido por las personas que tratan de acceder al mercado de trabajo y quienes ya forman parte de éste. Todo ello se completa con un proyecto transnacional con socios de otros países europeos y un plan de acción.

${ }^{2}$ IESE Family Responsible Employer Index (Índice IFREI de Empresas Familiarmente Responsables).
} 
sector (servicios), el tamaño (grandes empresas) así como el porcentaje de mujeres y su situación de desigualdad en la plantilla, favorecen la existencia de programas de conciliación e igualdad de oportunidades (Chinchilla y León, 2011); (Chinchilla et al., 2007) Asimismo, la flexibilidad horaria está correlacionada con la innovación y el compromiso del empleado (Baltes et al., 1999); (León, 2010). Sin embargo, una de las conclusiones más importantes de estos trabajos es la de que, para que los programas de conciliación de las empresas funcionen, además de su permanencia en el tiempo, resulta decisivo el aspecto cultural, tanto en la empresa (Rothausen, González, Clarke, O_Dell), como en el entorno reforzado por la existencia de leyes y convenios (Meyer y Rowan, 1977).

En efecto, aunque las normas no pueden de facto resolver los problemas que se encuentran en terrenos más operativos, sí marcan directrices y garantizan unos mínimos que favorecen un cambio cultural profundo que, aunque lento en su desarrollo, se debe mantener constante en el tiempo (Meyer y Rowan). En el caso de la empresa, si no hay legislación e incentivos para diseñar planes de igualdad y conciliación o, por el contrario, si no se penaliza su incumplimiento, parece no asumirse por completo la necesidad de cambio y de actuación.

España además, posee una idiosincrasia que la hace diferente al resto de países del mundo (Den Dulk, 2001) y que hace que el contexto anterior sea especialmente relevante. En efecto, el desencuentro de horarios entre los miembros de la familia, las largas jornadas laborales y una flexibilidad mal dirigida (que puede ser la causa de la invasión del trabajo sobre la intimidad $)^{3}$ provocan un efecto negativo sobre la familia y frenan la aceptación e implementación del programa a largo plazo (Den Dulk, 1999).

El apoyo visible de la dirección, la participación masiva y social de empleados y de los comités de empresa, la necesidad de cambio social y una adecuada comunicación interna apoyada por una opinión pública favorable (Ryan y Kossek, 2008) son elementos nucleares del triunfo. En este sentido, cabe destacar la importancia de los medios de comunicación en la sensibilización (Papí y Cambronero, 2011) y cabe hablar así de la relevancia de la simbiosis entre comunicación interna y externa. Comunicar adecuadamente en la empresa evita la percepción de injusticia o la impresión de que el programa es una moda o una imposición (Glass y Fujimoto, 1995).

Por ello, el modo de informar sobre las políticas y más concretamente sobre el programa, tanto a nivel colectivo como individual, es muy importante en la negociación supervisor-empleado (Poelmans y Andrews, 2001), en la negociación colectiva y en la

\footnotetext{
${ }^{3}$ Las demandas profesionales intempestivas y fuera de horario, las reuniones a última hora de la tarde o la sobrecarga de trabajo son también síntomas de falta de control sobre el trabajo flexible. Por este motivo, algunos autores hablan de que con la flexibilidad se hacen más borrosos los límites entre vida privada y profesional y abogan por una flexibilidad moderada y bajo ciertas condiciones, defendiendo incluso la predictibilidad del horario en pro del respeto a la rutina familiar y a la necesidad de mantener unos ciertos límites entre trabajo y familia.
} 
demanda de acción individual, corporativa o social. Así, por ejemplo, algunos autores destacan que en muchos casos los empleados no conocen sus derechos y por ello no solicitan la flexibilidad (Still y Strang, 2003).

El punto anterior invoca una de las premisas básicas de la agenda research: que, en general, existe una sintonía constante entre los temas públicos, tanto a nivel personal como empresarial, y los temas mediáticos y políticos. En las sociedades de la información, la historia de los problemas sociales, en muchos casos personales, es la historia de su "ciclo de atención". Y la historia de los "ciclos de atención" se relaciona con la historia de los “ciclos de noticiabilidad" (Martín Llaguno, 2000, 2000a y 2002). La incorporación de un tema en el debate periodístico parece una condición indispensable para su consideración como problema público y para su futura resolución. En el proceso de formulación e implementación de políticas y respuestas sociales, los medios de comunicación colectiva, determinados sectores políticos y algunos grupos de presión, incluido los empresarios, juegan un papel nuclear (Milio, 1989; Walt, 1994).

Así, el interés de este trabajo se centra en explorar, desde un planteamiento integrador, el proceso de construcción del problema de la conciliación familiar y laboral, a partir de tres esferas: la parlamentaria, la periodística y la empresarial. El objetivo es observar la interrelación entre leyes, noticias y cultura corporativa, y analizar en qué medida funciona cada uno de estos tres aspectos como palanca de los demás.

Nos situamos en el periodo 2003-2007, periodo del "milagro económico español", caracterizado por la bonanza y el crecimiento de los índices de empleo femenino, y ubicado entre la promulgación del IV Plan para la Igualdad y la promulgación de la Ley Orgánica 3/2007 para la Igualdad Efectiva de Mujeres y Hombres (LOIEMH). Periodo, por tanto, del incremento fulgurante de las tasas de empleo femenino. La elección de este periodo responde a que existen estudios en otros países, que apuntan a que el debate en torno a la conciliación se aviva e inicia al tiempo que se produce un crecimiento de las tasas de empleo femenino.

En efecto, en España la incorporación de la mujer al trabajo ha sido lenta pero significativa. El empleo femenino ha ascendido hasta situarse cerca de los objetivos marcados por la estrategia de Lisboa, que situaba el mismo en torno al $60 \%$ en el año 2010, con un incremento de 7 puntos porcentuales en una década, del $51.1 \%$ en 1997 a $58.3 \%$ en $2007^{4}$.

Nuestra hipótesis de partida es que las normativas impelidas por el marco de la UE irrumpen en estos momentos en un tejido empresarial con poca sensibilidad hacia la conciliación y con culturas poco igualitarias en las empresas (Poelmans, Chinchilla y Cardona, 2003). El porcentaje de empresas con un programa de conciliación pasa de un $7 \%$ del total de las encuestadas en el 2003 a un $12 \%$ en el 2007, año en el que el horario

\footnotetext{
${ }^{4}$ Comisión de las Comunidades Europeas, Comunicación de la Comisión al Consejo, al Parlamento Europeo, al Comité Económico y Social Europeo y al Comité de las Regiones, Igualdad entre Hombres y Mujeres en el 2009.
} 
flexible, trabajo a tiempo parcial, semana o jornada comprimida, excedencia, vacaciones flexibles y teletrabajo muestran un mayor crecimiento en cuanto a uso por parte del empleado. Sin embargo, raramente estas políticas aparecen cohesionadas entre sí, formando un programa, pues crecen aisladamente en la anarquía. De nuevo, la figura del departamento de Recursos Humanos y la mayor presencia y tradición sindical en el sector de Producción, se muestran como decisivas en todo el proceso de cambio.

No obstante, el marco legal impulsa la cobertura del tema por los medios de comunicación y la consiguiente generación de un clima de opinión sensible hacia estos temas cala en algunas instituciones que, en el contexto de la creciente feminización del mercado laboral, empiezan a incrementar su presupuesto destinado a la implantación de políticas de flexibilidad en forma de programa, que incluye asesoramiento al empleado y formación en estos temas, consiguiendo con ello que su cultura "familiarmente responsable" mejore. Y esta mejora de la cultura corporativa se ve reflejada en el aumento del interés mediático y político hacia el conflicto familiar y laboral y las políticas de conciliación.

De esta manera, la retroalimentación entre las tres agendas -mediática, legislativa y empresarial- empieza a perfilarse como esencial, en lo que a nuestro juicio es la construcción y la resolución de un tema social. Retroalimentación que, sin embargo, queda suspendida por la irrupción de la crisis.

\section{Metodología}

Para acometer nuestro objetivo y comprobar nuestra hipótesis, hemos de operacionalizar en variables algunas dimensiones del contexto del problema social de la conciliación familiar y laboral. Para la prominencia política del tema, operacionalizaremos al variable iniciativas parlamentarias. Para la prominencia pública, las variables noticias y cultura empresarial y para la prominencia empresarial, las variables asesoramiento, formación y flexibilidad. Las fuentes: actas, noticias y respuestas de cuestionarios de empresas. Veamos:

\subsection{Actas}

Se compilan todas las iniciativas parlamentarias en las que han aparecido los términos conciliación/conflicto de la vida familiar y laboral en la VII Legislatura, concretamente de abril 2003 a abril 2004; y en la VIII Legislatura, concretamente de abril 2003 a abril de 2007. Estos datos se obtienen a partir de la consulta a la página web del 
Congreso de los Diputados 5 . Suman un total 122 iniciativas. Una vez acopiados los documentos, se codifican de acuerdo a un protocolo que recoge información sobre algunas coordenadas del texto: año, mes y legislatura de publicación, órgano parlamentario donde tiene lugar la sesión, entre otras. Y, con base en este protocolo, se agrupan las iniciativas por años y se realiza un estudio descriptivo basado en la serie temporal generada por las frecuencias absolutas utilizando el SPSS-18.5.

\subsection{Noticias}

Se recogen también todas las noticias sobre el conflicto/la conciliación familiar y laboral publicadas entre marzo de 2003 y diciembre de 2003 o 2007, en los 3 diarios de mayor tirada nacional según la Oficina de Justificación de la Difusión: El País, ABC y El Mundo. Se incluyen todas informaciones publicadas en la edición nacional impresa, cuyo titular o contenido contemplen uno o varios de los siguientes términos: conciliación, igualdad, conflicto familiar, conflicto laboral, balance laboral. La obtención de los textos se realiza mediante búsqueda digital en la página web y servicios de documentación de los periódicos El País, ABC y El Mundo. Se obtienen en 198 noticias que se agrupan en una base de datos por años. Con la variable se realiza un estudio descriptivo basado en la serie temporal generada por las frecuencias absolutas utilizando el SPSS-18.5.

\subsection{Encuestas}

La base de datos "Empresas" referente a la implantación de medias de conciliación e igualdad en las empresas se obtiene reuniendo los resultados de diversos estudios realizados durante el periodo que va del año 2003 al año 2007. Contiene información de 4.781 entidades, 3.285 PYMES y 1.496 grandes empresas. Sus resultados agregados son la base del Índice IFREI (IESE Family Responsible Index).

A partir de un cuestionario previamente validad ${ }^{6}$ esta base de datos cuenta con respuestas acerca de la accesibilidad, disponibilidad y uso por parte del empleado de treinta y una políticas referidas a flexibilidad horaria y espacial, asesoramiento, beneficios y formación; así como otros aspectos que definen la cultura directiva y la organización en

\footnotetext{
${ }^{5}$ Véase: http://www.congreso.es. Esta página constituye una base de datos útil para el análisis de políticas públicas, por su capacidad de aglutinar información de interés para la realización de estudios retrospectivos (cuenta con datos desde el período constitucional establecido tras la dictadura franquista), de fácil acceso, transparente y aceptablemente actualizada.

${ }^{6}$ El impacto del estudio IFREI del IESE en las iniciativas de la Administración Pública ha sido notable, tanto en la publicación de guías de buenas prácticas por parte de la Comunidad de Madrid (años 2005, 2006 y 2007) como por la Generalitat de Catalunya (2003, 2009). También aparecen premios como el de la Empresa Flexible organizado por la consultora CVA desde el año 2002 y certificaciones como la impulsada por la Fundación Más Familia. En el ámbito académico el primer paper sobre este tema desde el punto de vista de las empresas se publica en el año 2003 (Poelmans, Chinchilla y Cardona, 2003).
} 
el modo de implantarse esas políticas. Para este artículo se incluyen 9 de las 31 preguntas referidas a las cuestiones seleccionadas: cultura, flexibilidad, asesoramiento y presupuesto. Veamos:

La cultura se define como un grupo de políticas de empresa, suficientemente relevantes y complementarias a las consideradas tradicionales dentro del ámbito de recursos humanos, cuya finalidad es ayudar al empleado a manejar con eficacia sus diferentes roles, como padre, cónyuge y trabajador, manteniendo siempre una filosofía de empresa acorde con esta decisión (Perry-Smith y Blum, 2000). Su operacionalización para este trabajo se ha realizado a partir de la media de las puntuaciones del cuestionario IFREI resultante del bloque formado por seis preguntas referidas a diversos hábitos ${ }^{7}$.

Por su parte, la flexibilidad (Bailyn, 1997) se ha entendido en sus tres dimensiones: temporal (bajo este concepto se instauran programas de trabajo flexible), operacional (que da lugar a procesos de trabajo flexible) y directiva (sensibilidad ante las demandas personales y familiares del empleado). Su operacionalización es el resultado de la media obtenida a partir de la disponibilidad de diferentes opciones en la empresa ${ }^{8}$.

La variable asesoramiento refleja el grupo de medidas enfocadas a la formación del empleado en materia de conciliación e igualdad así como el soporte dado a la familia y a la maternidad desde la empresa. El asesoramiento incluye educación y respaldo en la trayectoria profesional y personal-familiar. Diversos estudios confirman la asociación entre estas cuestiones: formación-asesoramiento y reducción del estrés (Wallace, 1997). Para este estudio ha sido operacionalizada a partir de la obtención de la media en las siguientes respuestas de la tabla? .

\footnotetext{
${ }^{7}$ Estas cuestiones son las derivadas de la aplicación de una escala que mide la disponibilidad de cuatro políticas: se espera que los empleados se lleven trabajo a casa, se juzga como menos comprometido con la empresa a quien se beneficia de un permiso o reducción de jornada por causa familiar, se anima a los empleados a irse a casa pasada cierta hora y las decisiones del departamento de personal tienen en cuenta la situación familiar/personal. Con formato de respuesta de cuatro puntos: $1=$ nada; $2=$ un poco; $3=$ bastante y $4=$ totalmente. Las respuestas medias en los distintos segmentos de la muestra se han comparado usando pruebas de hipótesis convencionales, $\mathrm{t}$ y ANOVA.

${ }^{8}$ Estas cuestiones son las derivadas de la aplicación de una escala que mide la disponibilidad de siete políticas: horario laboral flexible, trabajo a tiempo parcial, mediodía libre a cambio de alargar la jornada el resto de la semana, jornada reducida a cambio de una reducción salarial, excedencia para cuidar de un familiar, calendario de vacaciones flexible y posibilidad de trabajar desde casa. Con formato de respuesta de cuatro puntos: $1=$ ninguno; $2=$ algunos; $3=$ la mayoría y $4=$ todos. Las respuestas medias en los distintos segmentos de la muestra se han comparado usando pruebas de hipótesis convencionales, t y ANOVA.

${ }_{9}^{9}$ Estas cuestiones son las derivadas de la aplicación de una escala que mide la disponibilidad de cuatro políticas: asesoramiento profesional, asesoramiento personal/familiar, formación sobre cómo conciliar trabajo y familia y formación en gestión del tiempo y estrés. Con formato de respuesta de cuatro puntos: $1=$ ninguno; $2=$ algunos; $3=1$ la mayoría y $4=$ todos. Las respuestas medias en los distintos segmentos de la muestra se han comparado usando pruebas de hipótesis convencionales, $\mathrm{t}$ y ANOVA.
} 
Finalmente, el presupuesto se define como parte de una estrategia que busca un beneficio, se apoya en unos recursos económicos y humanos, y pretende lograr un objetivo en un tiempo determinado, asumiendo costes y también riesgos (Nah, Lau y Kuang, 2001). La adopción del programa depende en gran parte de que exista un presupuesto específico impulsado por un liderazgo fuerte (Powell y Mainiero, 1999). El marco legal de la Ley de Igualdad del año 2007 impulsó este aspecto ya que existieron ayudas y asesoramiento para implantar los programas también en PYMES, que antes no disponían de presupuesto para hacerlo. En esta investigación, hemos trabajado en esta variable a partir de la respuesta dada específicamente a esta cuestión dentro del cuestionario ${ }^{10}$.

“Además del deber general de las empresas de respetar el principio de igualdad en el ámbito laboral, se contempla, específicamente, el deber de negociar planes de igualdad en las empresas de más de doscientos cincuenta trabajadores o trabajadoras. La relevancia del instrumento de los planes de igualdad explica también la previsión del fomento de su implantación voluntaria en las pequeñas y medianas empresas [...] Para impulsar la adopción voluntaria de planes de igualdad, el Gobierno establecerá medidas de fomento, especialmente dirigidas a las pequeñas y las medianas empresas, que incluirán el apoyo técnico necesario" (España, 2007: 12613).

Como conclusión de este apartado metodológico, decir que se ha realizado un estudio de las respuestas medias en los distintos segmentos de la muestra. Se han comparado usando pruebas de hipótesis convencionales, t y ANOVA. En las pruebas de hipótesis, p designa el nivel de significación. Estos análisis se han realizado con Stata 10, menos el del modelo causal, para el que ha usado Mplus 5. Para la visualización de la retroalimentación entre las tres agendas observadas hemos realizado un gráfico de líneas temporales con el SPSS-11.5.

\section{Resultados}

El gráfico $\mathrm{n}^{\mathrm{o}} 1$ representa la evolución de el número de iniciativas parlamentarias presentadas en el Congreso en el tiempo acotado; el número de noticias publicadas en los diarios analizados, años 2003-2007; la evolución de la media de respuestas correspondientes a las preguntas sobre cultura, flexibilidad, asesoramiento; y finalmente, el volumen de presupuesto.

\footnotetext{
${ }^{10}$ Estas cuestiones son las derivadas de la aplicación de una escala que mide la fase en la que está el programa y el presupuesto asignado. La primera cuestión: las políticas familiarmente responsables, cuenta con un formato de respuesta de cuatro puntos: $1=$ no están en la agenda; $2=$ fase inicial; $3=$ poco utilizadas y $4=$ muy utilizadas. La segunda cuestión: presupuesto asignado, cuenta con un formato de respuesta de otros cuatro puntos: $1=$ ninguno; $2=$ eventual; $3=$ específico y $4=$ revisión anual. Las respuestas medias en los distintos segmentos de la muestra se han comparado usando pruebas de hipótesis convencionales, t y ANOVA.
} 
Gráfico 1. Evolución de las iniciativas parlamentarias; las noticias publicadas; la flexibilidad, la cultura, el presupuesto y el asesoramiento disponible en las empresas españolas.

Años 2003-2007.

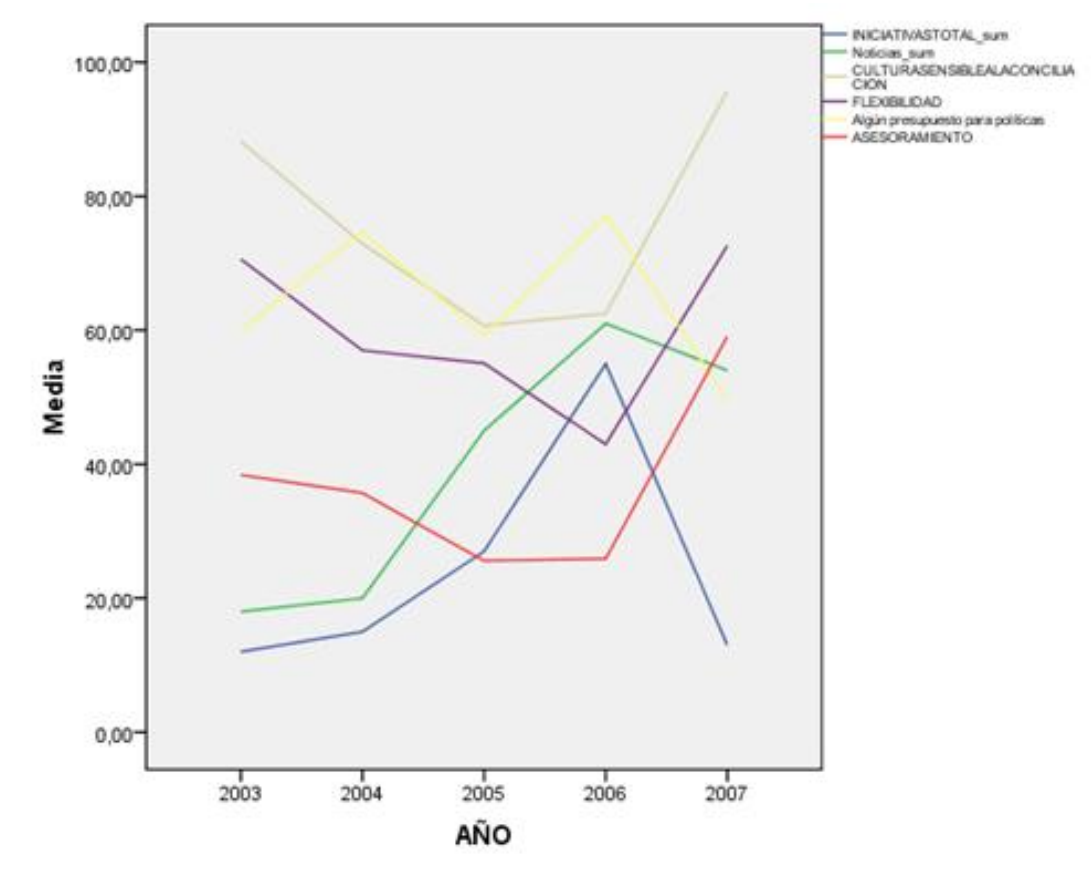

Fuente: elaboración propia.

Tal como se observa con relación a las iniciativas, en el año 2003, han transcurrido cinco años desde la promulgación de la Ley 39/1999, de 5 de noviembre, para promover la Conciliación de la Vida Familiar y Laboral de las personas trabajadoras, y el interés político por esta cuestión no es alto, hecho que queda reflejado en el número de intervenciones sobre el tema en la cámara. En el año 2004 el número de intervenciones relacionadas con el problema empieza a remontar. El tema no está en la agenda política de un modo claro hasta 2005-2006, años previos a la Ley de Igualdad de Oportunidades, y sufre un brusco descenso en el año 2007, ya muy cerca del inicio de la crisis económica.

Por su parte, respecto a las noticias, y después del interés inicial por el tema provocado por la promulgación de la Ley 39/1999, para promover la Conciliación de la Vida Familiar y Laboral de las personas trabajadoras; la cobertura en 2003, como sucede con el interés político, decae. No en vano, como ponen de manifiesto algunos trabajos (Martín Llaguno, Beléndez y Hernández, 2007); (Llaguno y Hernández, 2010); estamos asistiendo hasta el 2004 al fin del ciclo inicial de interés generado por la promulgación Ley de Conciliación. Este ciclo había comenzado vinculado a la primera legislatura del PP y se cierra en abril de 2004 con el cambio de Gobierno. 
Precisamente a partir de este momento, marzo de 2004, empieza a aumentar la atención mediática que cuenta con las cotas más altas de cobertura del conflicto en 2006. En este periodo, este tema no sólo crece en los debates parlamentarios, como hemos visto, sino en los debates sindicales y, como se aprecia, en los discursos periodísticos (Gómez, 2003); (Añino, Aragón y Rocha, 2009); (Gallardo, 2009); (Escudero, 2008); (Lousada Arochena, 2008); (Roldán, 2010); (Valdés y Sobrino, 2011). Son los años del crecimiento económico de España y de la incorporación masiva de la mujer al ámbito laboral, la mayor de Europa. Precisamente, la aprobación del Consejo de Ministros del anteproyecto de la Ley Orgánica para la Igualdad Efectiva de Mujeres y Hombres (LOIEMH) provoca que, en marzo de 2006, se haga la cobertura más extensiva del tema en especial en el diario El País. En el marco de esta acción legislativa, gobierno, oposición, Comunidades Autónomas y ciudadanos particulares exponen sus posturas sobre un documento que "obliga a las Pymes a negociar planes de igualdad" y que considera a los empresarios como "actores decisivos en el tema". La oposición considera que esta política va contra la libertad, al igual que la CEOE, mientras que los sindicatos consideran que las medidas son insuficientes. Los permisos de paternidad recogidos en este documento y los horarios flexibles tienen el mayor impacto mediático.

Pero, pasado este alud informativo y parlamentario, la tendencia cambia. En 2006, el año previo a la crisis económica, la cuestión empieza a relegarse en este contexto a un segundo plano en la esfera pública.

¿Qué sucede mientras tanto en las empresas? Para responder a esta pregunta nos fijamos que las tres variables analizadas: cultura, flexibilidad y asesoramiento siguen una tendencia descendente desde 2003 hasta 2006. En 2004 y 2005, más de un lustro después de que se promulgue la Ley de Conciliación, en los años de mayor presencia femenina en el mercado laboral, la sensibilidad familiarmente responsable va bajando en picado.

Una posible explicación coyuntural es que en su etapa inicial, la Ley de Conciliación de 1999 (Poelmans, Chinchilla y Cardona, 2003) había empujado a incluir en convenios colectivos un largo elenco de prestaciones sociales nuevas, como permisos, reducciones de jornada y excedencias. Sin embargo la disponibilidad de estas medidas habría ido decayendo a medida que la visibilidad pública de la norma y los presupuestos y subvenciones hubieran ido disminuyendo. La sensibilidad habría repuntado a partir de 2006 (Chinchilla y León, 2011), al amparo de la promulgación de la Ley de Igualdad, ante promesas de ayudas y subvenciones económicas para elaborar planes de igualdad $^{11}$, así como de las posibles ventajas para las empresas y en concursos públicos respecto a la contratación de mujeres. De hecho, en este año, se produce una inflexión. Se reactiva la sensibilidad de las empresas por la conciliación y, a partir de ese momento, empieza a aumentar el porcentaje de instituciones que dicen contar con

\footnotetext{
${ }^{11}$ Convocatoria de ayudas del Ministerio para elaborar planes de Igualdad en las empresas, véase: http://www.msc.es/eu/ssi/igualdadOportunidades/iEmpleo/Planes_de_Igualdad/conv_pdi.htm Ley de igualdad y su impacto en empresas, véase: http://www.sondeoeconomico.com/2011/06/16/ley-de-igualdad-y-su-impacto-en-empresas/ 5.100 Inspecciones programadas a empresas para vigilar si cumplen igualdad, veáse: http://www.expansion.com/agencia/efe/2011/07/01/16299999.html
} 
algún presupuesto asignado al fomento de políticas de flexibilidad (Sánchez, Mayo y Chinchilla, 2006).

En cambio, la variable asesoramiento ofrece resultados absolutos muy bajos a lo largo de periodo. Una vez más, la implantación real de la flexibilidad está muy por debajo de los planes de acción cuyo objetivo es gestionar el cambio. El motivo es que los directores de recursos humanos suelen quedarse en un nivel de detección, sensibilización y diseño del programa, pero les cuesta más orquestar planes de asesoramiento y formación que supongan un presupuesto definido y un plan de acción con la consiguiente traducción del mismo en formación profesional, familiar y sobre gestión del tiempo (Miliken, Martins y Morgan, 1998).

Así, la cultura empresarial habría sido reactiva a las normas. La tradición de largas jornadas laborales y baja productividad, siendo estilos organizativos poco productivos y denunciados por plataformas ciudadanas de diverso tipo (León, 2005), habría permanecido latente. Como pone de manifiesto el hecho de que durante estos años, el porcentaje de empresas denominadas excelentes según el índice IFREI tan sólo evoluciona del 7 al 12\% hasta el 2005, para después volver a bajar partir del $2006^{12}$.

\section{Discusión}

En este trabajo se partía de la hipótesis de que para la construcción social del problema de la conciliación, en España se produce un mimetismo entre leyes, noticias, cultura y actuaciones familiarmente sensibles en las empresas.

Así, esta investigación plantea que, en efecto, parece haber una sintonía entre iniciativas parlamentarias e informaciones que, coordinadamente, podrían haber provocado en diferido una presión en las repuestas empresariales a través de mecanismos directos, como los económicos, e indirectos, como los cambios en el clima de opinión. Aunque es difícil valorar los niveles de sensibilización ciudadana generados por las agendas políticas y periodísticas (puesto que no existe un mecanismo que registre sistemáticamente la opinión de los ciudadanos con respecto al conflicto y la conciliación familiar y laboral a lo largo del tiempo) a través de otros trabajos (Guirao y Martín Llaguno, 2012) y de los indicadores manejados, se constata en el tiempo el proceso de construcción de un problema social. En un escenario teóricamente favorable, bonanza económica y, por tanto, un profundo proceso de feminización del mercado laboral, no se logran, sin embargo, cambios radicales respecto al porcentaje de mujeres de la población activa, respecto a la tasa de fecundidad, respecto al acceso femenino a puestos directivos y respecto a la organización flexible del trabajo sin recelos por parte de las empresas (Martín Llaguno, Beléndez Vázquez y Hernández Ruiz, 2007). Parte del problema podría derivar de que la conciliación "laboral y familiar", como herramienta para la igualdad, se

12 Sin embargo la ley de Igualdad del 2007 hará remontar de nuevo esta tendencia hasta el 12\%. 
presenta como un "artefacto" de la política internacional desde el 1995 para la aceleración de la incorporación de la mujer a un mercado de trabajo cada vez más ávido de mano de obra flexible (Martín Llaguno y Guirao Mirón, 2012) y no como la institucionalización de un problema de los ciudadanos.

Sea con el enfoque que fuere, nuestro estudio pone de manifiesto que, como en otros países, las distintas presiones generadas en este proceso de construcción social, especialmente si tienen dimensiones económicas, podrían ser desencadenantes importantes en la implantación de las políticas corporativas (DiMaggio y Powell, 1983). Y este hecho es ya una aportación, puesto que trabajos previos habían descartado en España la eficiencia de distintas presiones, por encontrarse la conciliación familiar y laboral en fases muy iniciales del proceso de institucionalización (Poelmans, et al., 2003 y 2003a).

Por otra parte, y tal y como se refleja en otros trabajos internacionales (Korabic, Lero y Ayman, 2003), en nuestro país se cumple la tendencia de que en las naciones que se impulsa legislación en materia de igualdad, mejora también la cultura familiarmente responsable de las empresas. De hecho, Blau (1964) en su Teoría del intercambio social compara la dinámica interna de la empresa con otras instituciones u organismos sociales, en los que se dan intercambios, pactos y compromisos que son impulsados por el contexto legal favorable y suponen una mayor identificación del empleado con la misión de la empresa. Tres décadas más tarde Organ (1990) mantiene este punto de vista, partiendo de las aportaciones de Homans (1958) y Thibaut y Kelley (1959). Algunos autores hablan más tarde de la presión institucional y legal, en la toma de decisiones directivas, respecto al diseño de políticas corporativas de conciliación e igualdad en las empresas (Tolbert y Zucker, 1983).

Hasta la fecha, la literatura académica había recurrido a la teoría institucional para el estudio de las diferentes causas en la implantación de planes de conciliación (Goodstein, 1995; Ingram y Simons, 1995). Esta teoría pone el énfasis en las estructuras, procesos y reglas sociales que llegan a incidir internamente en las empresas, en los mandos directivos y en la cultura hasta llegar a definir los programas. Esta concepción de la cultura de empresa ha dado como resultado un buen número de estudios conceptuales y empíricos sobre desarrollo organizativo. En ellos queda patente que el temor al cambio organizativo resultante de la flexibilidad horaria y del teletrabajo, constituye la principal presión institucional a la implantación de un programa de conciliación (Dimaggio y Powell, 1983). Por este motivo, en opinión de algunos autores, estos programas constituyen un auténtico reto para las organizaciones más progresistas (Bielby y Baron, 1986). Siendo las empresas pioneras quienes los implantan (Galinsky y Stein, 1990). Además, una cultura corporativa conciliadora es capaz de estimular o mejorar los valores más competitivos de la organización y del mercado, como por ejemplo, productividad y eficacia en el lugar de trabajo (Quinn y Rohrbaugh, 1983); (Gonyea y Googins, 1992).

En definitiva, en este trabajo, se incide en la importancia de las influencias internas y externas en las decisiones directivas corporativas, que llevan a la generalización de los programas. Ningún trabajo había abordado esas fuerzas desde un planteamiento de 
establecimiento de la agenda. Creemos que esta aproximación puede ser un marco conceptual apropiado para valorar y medir factores que influyen en la disminución o el acrecentamiento del conflicto familiar y laboral. Máxime si se considera esta opción como prolongación o complemento de la propuesta de Bronfenbrenner (1976), quien considera que la conducta humana se desarrolla como fruto de la interacción del nivel micro, meso y macro. La persona y la familia constituiría el nivel micro, la empresa el nivel meso y la sociedad el nivel macro.

Martín Llaguno et al., (2011) siguiendo a Poelmans y Andrews (2001), afirman que el análisis de la conciliación desde un exclusivo foco en el primero de los niveles es prolijo. En efecto, el conflicto familia y trabajo empieza a captar la atención de la comunidad científica al inicio de la "era de la información" cuando, con la incorporación masiva de la mujer al mercado laboral, la interconexión entre el mundo productivo y reproductivo empieza a hacerse cada vez más clara. La investigación de esta nueva problemática avanza en la primera década de manos de la psicología y se centra originariamente en cuestiones relacionadas con la experiencia de estos problemas por parte de los individuos (nivel micro). Como esquematiza Poelmans y Andrews (2001), los estudios de este tipo, con una base teórica relativamente consistente, se interesan por aislar, por una parte, los factores personales que acrecientan o moderan este tipo de problemas; y, por otra, las repercusiones de estos conflictos sobre la salud individual y familiar.

Posteriormente, a principios de los 90, una vez obtenidos resultados en el campo anterior, empiezan a generarse otro tipo de investigaciones centradas ya, no en la experiencia personal de estos problemas, sino en la experiencia corporativa y en las soluciones propuestas desde esta esfera para los mismos (nivel meso). Estas investigaciones son mucho más escasas que las anteriores por una razón puramente coyuntural: los programas de conciliación se empiezan a implantar especialmente a finales de los 80 en la sociedad, y a principios de los 90 en algunas empresas, por tanto, ha sido en las últimas décadas cuando se han empezado a poder evaluar. De nuevo estos estudios se centran, en primer lugar, en las circunstancias que hacen implantar este tipo de políticas; en segundo lugar, en los beneficios que estas medidas reportan y, en tercer lugar, en el análisis de las repercusiones de medidas de conciliación concretas. En este contexto se enmarcaría el estudio IFREI.

Finalmente, en esta década empiezan a aflorar con fuerza los planteamientos de estudios que pretenden valorar y evaluar el impacto de las políticas públicas, o alguna de sus dimensiones, para la erradicación del conflicto familiar y laboral y la promoción de la conciliación como factor de igualdad. Como se verá, estos trabajos son muy recientes, se ubican sobre todo en Europa y, partiendo casi siempre de la revisión de la literatura gris, se centran en la realización de comparaciones críticas entre tres factores fundamentales, primero, los sistemas de protección social en materia de conciliación; en segundo lugar, el análisis de los enfoques o los marcos discursivos sobre el problema en la legislación; y en tercer lugar, las repercusiones de normativas concretas sobre ejes de actuación, como los convenios colectivos o la comunicación institucional. Sin embargo, escasean totalmente los estudios multinivel 
(macro-meso-micro), que parten de modelos que integren el conflicto/la conciliación como dos caras de un mismo problema.

El trabajo presentado es fruto de dos proyectos de investigación distintos:

1- El proyecto $\mathrm{I}+\mathrm{D}+\mathrm{I}$ "La construcción social del conflicto familia trabajo. Análisis mediático, político y de la realidad" financiado por el Instituto de la Mujer en la convocatoria 2007, cuyo objetivo era analizar la construcción del discurso público en torno a un tema "emergente": el problema del conflicto familia-trabajo y su solución en la conciliación de la vida familiar, en la última década. Dicha investigación integra un estudio de la agenda política, iniciativas y leyes; la agenda mediática, análisis de prensa; y el impacto en la realidad social, encuestas de usos del tiempo EET y población activa, EPA.

2- El proyecto IFREI (Iese Family Responsible Index) cuya edición anual durante este periodo constituyó un índice de clima en las empresas, obtuvo financiación de proyectos Equal de la UE, el Ministerio de Trabajo y Asuntos Sociales, así como el Ministerio de Igualdad y las correspondientes Consejerías de Empleo de la Comunidad Autónoma de Madrid y de la Generalitat de Catalunya. El objetivo era medir el grado de implantación real, de uso por parte del empleado, respecto a las políticas de flexibilidad aprobadas en convenio y vigentes por ley. A esta información descriptiva de la situación real, en el seno de las empresas se añadían otros factores como el rol del directivo, el presupuesto asignado, y la cultura de empresa respecto a horarios y promoción profesional dentro de las compañías.

La diversidad de fuentes ha supuesto adaptar las variables y mediciones para realizar comparaciones que permitieran captar el estado de la cuestión, pero la oportunidad de comparar resultados sólidos en torno a un mismo problema era única. No obstante, queda pendiente para futuros estudios realizar análisis estadísticos y mediciones que permitan valorar cuantitativa y cualitativamente las influencias entre elementos.

En este sentido el reto más importante es el de operacionalizar conceptos y utilizar variables estandarizadas, que posibiliten comparaciones fiables para valorar el efecto de las políticas y normativas públicas, de una forma directa, sobre el conflicto familiar y laboral. Hasta ahora, los trabajos existentes valoran las relaciones indirectas de las políticas con la inserción de la mujer en el mercado de trabajo, con las tasas de natalidad, con la remuneración en función de género, etc... Pero no miden la incidencia real sobre una variable cuantificada desde hace años, desde la psicología organizacional. Sería importante incluir en un ulterior estudio el conflicto familiar y laboral del empleado, para ver cómo incide en él el contexto social y las política organizacionales. 


\section{BIBLIOGRAFÍA}

- Aguirre, Rosario y García Sainz, Cristina (2005): El tiempo, los tiempos, una vara de desigualdad. Naciones Unidas, Comisión Económica para América Latina y el Caribe (CEPAL).

- Añino, Sara; Aragón, Jorge y Rocha Fernando (2009): Los planes de igualdad entre mujeres y hombres en la negociación colectiva. Madrid: Instituto de la Mujer.

- Bailyn, Lotte (1997): “The impact of corporate culture on work-family integration”. En: Parasuraman y Greenhaus (eds.): Integrating work and family; challenges and choices for a changing world. Westport, CT: Quorum Books, pp. 209-219.

- Baltes, Boris et al. (1999): "Flexible and compressed workweek schedules. A metaanalysis of their effects on work-related criteria". En: Journal of Applied Psychology, n 84, pp. 496-513.

- Beer, Michael et al. (1990): “Why change programs don't produce”. En: Harvard Business Review, vol. 68, nº 6 , pp. 158.

- Bielby Willian T. y Baron, James N. (1986): "Men and women at work: sex segregation and statistical discrimination”. En: American Journal of Sociology, vol 91, nº 4, pp. 759-799.

- Blau, Peter (1964): Exchange and power in social life. New York: Wiley.

- Bronfenbrenner, Urie (1976): "The ecology of human development: history and perspectives". En: Psychologia, vol. 19, nº 5, pp. 537-549.

- Chinchilla, Núria; et al. (2007): Análisis sectorial de las políticas de conciliación. Conclusiones del estudio IFREI 2006 basado en 360 empresas. España: IESE-CITF.

- Chinchilla, Núria y León, Consuelo (2005): Empresas Familiarmente Responsables (EFR).

La comunicación empresarial y la gestión de los intangibles en España y Latinoamérica. España: Pirámide, pp. 225-234.

. (2011): Diez años de la conciliación en España. Editorial Grupo 5, pp. 1-161.

- Chinchilla, Nuria; Poelmans, Steven; León Consuelo (2003): "Políticas de conciliación trabajo-familia en 150 empresas españolas". En: IESE, D/498.

- Clarke, Liz (1994): The essence of change. The essence of management series. New York: Prentice Hall.

- Covin, Teresa Joyce y Kilmann, Ralph (1990): "Participant perceptions of positive and negative influences on large-scale change". En: Group \& Organization Studies, vol. 15, n², pp. 233-248.

- Del Campo, Salustiano (1990): “La familia simétrica y el trabajo de la mujer”. En: Anuales de la Real Academia de Ciencias Morales y Políticas, no 67, pp. 313-320.

- Den Dulk, Laura (1999): Employers and parental leave: A comparative analysis. Brussels: Ed. Peter Moss \& Fred Deven, NIDI/CBGS Publications.

(2001): Work-family arrangements in organizations: Across-national study in the

Netherlands, Italy, the United Kingdom, and Sweden. Amsterdam: Rozenberg Publishers. 
- Dimaggio, Paul y Powell, Walter (1983): "The iron cage revisited: Institutional isomorphism and collective rationality in organizational fields". En: American Sociological Review, n 48, pp. 147-160.

- Duck, Jeanie D. (1993): “Managing change: The art of balancing”. En: Harvard Business Review, vol. 71, nº 6, pp. 109.

- Durán, M. Ángeles (1997): "La investigación sobre el uso del tiempo en España: algunas reflexiones metodológicas”. En: Revista Internacional de Sociología, $\mathrm{n}^{\circ} 18$, septiembrediciembre, pp. 163-193.

(2003): "El trabajo no remunerado en la familia". En: Arbor, no 694, pp. 239-268.

- Escudero, Ricardo (2008): "Márgenes y retos de la negociación colectiva tras la ley orgánica de igualdad efectiva entre mujeres y hombres". En: Ricardo Escudero (ed.): La Negociación Colectiva en España una mirada interdisciplinar. Madrid: Cinca, pp. 178-200.

- España (2007): "Ley Orgánica 3/2007, de 22 de marzo, para la igualdad efectiva de mujeres y hombres", [en línea] Disponible en: http://www.boe.es/boe/dias/2007/03/23/pdfs/A1261112645.pdf [12/01/2013].

- Galinsky, Ellen y Stein, Peter (1990): "The impact of human resource policies on employees". En: Journal of Family Issues, n¹1, pp. 368-383.

- Gallardo Moya, Rosario (2009): "Los nuevos planes de igualdad en la empresa: una análisis de las primeras experiencias". En: Revista de Derecho Social, n 48, pp. 83-107.

- Glass, Jennifer y Fujimoto, Tetsushi (1995): "Employer characteristics and the provision of family responsive policies". En: Work and Occupations, vol, 22, n 4, pp. 380-411.

- Gómez, Sandalio (2003): “La negociación colectiva. Reformas pendientes”. En: Carlos de Benito (ed.): Relaciones laborales. Asignaturas pendientes. Madrid: Editorial Pearson Educación, pp. 100-125.

- Gonyea, Judith y Googins, Bradley (1992): "Linking the worlds of work and family: beyond the productivity trap". En: The Human Resource Management, vol. 31, no 3, pp. 209226.

- Goodstein, Jerry (1994): "Institutional pressures and strategic responsiveness: Employer involvement in work-family issues". En: Academy of Management Journal, $\mathrm{n}^{\circ}$ 37, pp. 350382.

- Guirao Mirón, Cristina y Martín Llaguno, Marta (2012): "El marco jurídico, mediatico y politico de la conciliación en España. Desde 1999 a 2009”. En: Estudios sobre el mensaje periodístico, vol. 18, número especial octubre 2012, pp. 475-484.

- Homans, George (1958): "Social Behaviour as Exchange". En: American Journal of Sociology, $\mathrm{n}^{\mathrm{o}}$ 6, p. 63.

- Iglesias de Ussel, Julio (1995): “Trabajo y familia en España”. En: Revista Internacional de Sociología, $\mathrm{n}^{\circ} 11$, pp. 171-198.

. (2004), "Familia y trabajo de la mujer". En: Arbor, no 702, pp. 167-185. 
- Ingram Paul y Simons, Tal (1995): "Institucional and resource dependence determinants of responsiveness to work and family issues”. En: Academy of Management Journal, vol. 38, $\mathrm{n}^{\circ}$ 5, pp. 1466-1482.

- Korabic, Karen; Lero, Donna S. y Ayman, Roya (2003): "A multi-level approach to crosscultural work-family research: A micro- and macro-perspective”. En: International Journal of Cross Cultural Management, vol. 3, nº 3, pp. 289-303.

- Kraut, Allen I. (1990): "Some lessons on organizational research concerning work and family issues". En: Human Resource Planning, n 13 , pp. 78-89.

- León, Consuelo (2005): Racionalizar nuestros horarios, un reto necesario. Ed. Libro Blanco de la Comisión Nacional para la racionalización de los horarios españoles y su normalización con Europa. Fundación Independiente y Ministerio de Administraciones Públicas.

(2010): "Factores de éxito en la implantación de políticas de conciliación trabajo y familia en las empresas españolas (2003-2007)". Tesi doctoral, Universitat Politècnica de Catalunya, Departament d'Organització d'Empreses.

- León, Consuelo y Chinchilla, Núria (2007): Les millors pràctiques de conciliació treballfamília a l'empresa. Barcelona: Departament de Benestar i Familia. Generalitat de Catalunya.

- Lousada Arochena, José Fernando (2008): "El marco normativo de la negociación colectiva de medidas de igualdad de mujeres y hombres”. En: Revista de Derecho Social, no 41, pp. 3154.

- Martín Llaguno, Marta (2000): "De la detección al recuerdo: una historia periodística del sida". En: Zer: Revista de Estudios de la Comunicación, nº 8, pp. 41-66.

- (2000a): "La función de recuerdo de los medios. Las coberturas de 'grandes temas"”. En: Comunicación y Sociedad XIII, nº 1, pp.115-138.

- _ _ (2002): La función de recuerdo de los medios de difusión. ¿Qué pasa cuando en los medios parece no pasar nada sobre un tema? Alicante: Universidad de Alicante.

- Martín Llaguno, Marta; Beléndez Vázquez, Marina y Hernández Ruiz, Alejandra (2007): La mujer en las agencias de publicidad. Categorías, especializaciones y conflicto trabajofamilia en las agencias españolas. Madrid: Asociación Española Agencias de Publicidad.

- Martín Llaguno, Marta y Hernández Ruiz, Alejandra (2010): "Radiografía de los docentes en publicidad en España. Un estudio psicolaboral con enfoque de género". En: Pensar la publicidad: Revista Internacional de Investigaciones Publicitarias, vol. 4 nº 2, pp. 25-50.

- Martín Llaguno, Marta, Cristina Guirao Mirón, Marián Navarro Beltra, Dolores Frutos Balibrea, Marina Beléndez Vázquez Alejandra Hernández Ruiz (2001): "La construcción social del problema del conflicto trabajo-familia. análisis mediático, político y de la realidad". Proyecto I+D+I, Financiado por Instituto de la Mujer. Informe final NIPO: 685-12-035-9. Madrid: Instituto de la Mujer, [en línea] Disponible en: http://www.inmujer.es/areasTematicas/estudios/estudioslinea2012/docs/construccionSocialCon flicto.pdf [14/01/2013]. 
- Martín Llaguno, Marta y Guirao Mirón, Cristina (2012): "El debate parlamentario sobre conciliación familiar y laboral en España: análisis de una década de iniciativas”. En: Alternativas. Cuadernos de Trabajo Social, $\mathrm{n}^{\circ}$ 19, pp. 59-76.

- Meil, Gerardo (1997): "El papel de los niños en la redefinición del trabajo doméstico en la nueva familia urbana española”. En: Revista Internacional de Sociología, no 16, pp. 39-56.

- Meyer, John y Rowan, Briem (1977): "Institutionalized Organizations: Formal Structure as Myth and Ceremony”. En: American Journal of Sociology, vol. 83, n² 2, pp. 340-363.

- Milliken, Frances; Martins, Luis y Morgan, Hal (1998): "Explaining organizational responsiveness to work-family issues: the role of human resource executives as issue interpreters". En: Academy of Management Journal. Briarcliff Manor, Oct., vol. 41, n 5, pp. 580-592.

- Milio, Nancy (1989): Promoting health through public policy. Ottawa: Canadian Public Health Association.

- Nah, Fiona F. H.; Lau, Janet L. S. y Kuang, Jinghua (2001): "Critical factors for successful integration of enterprise systems". En: Business Process Management Journal, vol. 7, n 3, pp. 285-96.

- Organ, Dennis (1990): “The motivational basis of organizational citizenship behavior". En: Research in Organizational Behavior, $\mathrm{n}^{\mathrm{o}} 12$, pp. 43-72.

- Papí, Natalia, Cambronero, Belén (2011): “Acciones públicas de sensibilización de género.

El esfuerzo de la administración regional y local en comunicación publicitaria (1999-2007)".

En: Pensar la Publicidad: Revista Internacional de Investigaciones Publicitarias, vol. 5, $\mathrm{n}^{\mathrm{o}} 2$, pp. 181-203.

- Perry-Smith, Jill y Blum, Terry (2000): "Work-family human resource bundles and perceived organizational performance". En: Academy of Management Journal, vol. 43, n 5, pp. 1107-1117.

- Poelmans, Steven y Andrews, Wendy (2001): Launching flexible work-arrangements within Procter and Gamble EMEA.FH 716 (A) and FH 717 (B). Barcelona: IESE Publishing.

- Poelmans, Steven; Chinchilla, Núria y Cardona, Pablo (2003): "The adoption of familyfriendly HRM policies: competing for scarce resources in the labour market”. En: International Journal of Manpower Bradford, vol. 24, $\mathrm{n}^{\circ}$ 2, p. 128.

- Poelmans, Steven; Chinchilla, Núria y Cardona, Pablo (2003a): "Family-Friendly HRM Policies and the Employment Relationship". International Journal of Manpower, vol. 24, nº. 3, pp. 128-147.

- Powell, Gary y Mainiero, Lisa (1999): "Managerial decision making regarding alternative work arrangements". En: Journal of Occupational and Organizational Psychology, vol. 72, ${ }^{\circ}$ 1, pp. 41-56.

- Quinn, Robert y Rohrbaugh, John (1983): “A spatial model of effectiviness criteria: towards a competing values approach to organizational analysis". En: Management Science, vol. $29, n^{\circ} 3$, pp. 363-377. 
- Roldán, Aránzazu (2010): "Derechos colectivos y de participación de los trabajadores en materia de igualdad”. En: Ricardo Escudero Rodríguez (ed.): Empleo Público, Igualdad, Nuevas Tecnologías y Globalización, Madrid: Cinca, pp. 142-159.

- Ryan, Ann Marie y Kossek, Ellen Ernst (2008): "Work-life policy implementation: Breaking down or creating barriers to inclusiveness?". En: Human Resource Management, vol. $47, n^{\circ} 2$, pp. 295-310.

- Sánchez, Esther; Mayo, Margarita y Chinchilla Núria (2006): Guía para la igualdad de oportunidades entre mujeres y hombres en la empresa. Madrid: Consejería de Empleo y Mujer, Comunidad de Madrid.

- Still, Mary y Strang, David (2003): Institutionalizing family-friendly policies. Ithaca, NY. Cornell University Press: Ed. P. Moen, pp. 288-309.

- Thibaut, John y Kelley, Harold (1959): The social psychology of groups. New York.

- Tobío, Constanza (2005): Madres que trabajan: Dilemas y estrategias. Universidad Carlos III: Ed. Cátedra.

- Tobío, Constanza y Fernández Cordón, Juan Antonio (1999): "Monoparentalidad, trabajo y familia". En: Revista Internacional de Sociología, no 22, pp. 67-97.

- Tolbert, Pamela y Zucker, Lynne (1983): "Institutional sources of change in the formal structure of organizations: The diffusion of civil service reform 1880-1935". En: Administrative Science Quarterly, vol. 28, $\mathrm{n}^{\circ}$ 1, Mar., pp. 22-39.

- Valdés Dal-Ré, Fernando y Sobrino, Gemma (2011): Análisis de medidas y planes de igualdad en la negociación colectiva. Madrid: Observatorio de Medidas y Planes de Igualdad en la Negociación Colectiva, UGT.

- Vandermerwe, Sandra y Vandermarwe, Andre (1991): "Making strategic change happen". En: European Management Journal, vol. 9, n 2, pp. 174-181.

- Villafañe y Asociados (2005): Monitor Español de Reputación Corporativa (MERCO).

- Wallace, Jean (1997): "It's about time: A study of hours worked and work spillover among law firm lawyers". L: Journal of Vocational Behavior, vol. 50, n 2, April, pp. 227-24.

- Walt Gill (1994): Health policy: an introduction to process and power. Londres: Witwatersrand University Press. 